\title{
MOLECULAR DIVERSITY OF FRESHWATER FISHES OF BANGLADESH ASSESSED BY DNA BARCODING
}

\author{
Md. Sagir Ahmed ${ }^{1 *}$, Sujan Kumar Datta ${ }^{2}$ and Ayesha Akhter Zhilik ${ }^{1}$ \\ Department of Zoology, University of Dhaka, Dhaka1000, Bangladesh
}

\begin{abstract}
This study represents the comprehensive molecular identification of freshwater fishes of Bangladesh based on a fragment of the cytochrome c oxidase subunit I (COI) gene in the mitochondrial genome. A total of 315 mitochondrial COI barcode sequences were obtained from 153 species, 114 genera, 49 families and 16 orders of fishes. The mean length of the sequences was 652 base pairs. For all the samples, \%G was significantly lower compared to the other nucleotides and \%GC was lower compared to \%AT ( $p$-value $<0.05)$. Also, a significantly lower \%GC content was observed in second and third codon position compared to the first one in all the samples $\left(1^{\text {st }}>2^{\text {nd }}>3^{\text {rd }}, p\right.$-value $\left.<0.05\right)$. The average K2P distances within species, genera, families and orders were $0.38 \%, 7.02 \%, 12.75 \%$ and $18.68 \%$, respectively. The mean interspecific distance was 18 -fold higher than the mean intraspecific distance. The K2P neighbor-joining (NJ) trees based on the sequences generally clustered species according to their taxonomic position. A total of 12 species were newly recorded in Bangladesh. High efficiency in species identification were demonstrated in the present study by DNA barcoding, and concluded that COI sequencing can be used as an authentic identification marker for freshwater fish species.
\end{abstract}

Key words: Freshwater fishes, COI, DNA Barcoding, Genetic Diversity, Phylogeny

\section{INTRODUCTION}

Bangladesh is a deltaic country that emerged on the confluence of the three mighty river systems, Padma-Ganges, Jamuna-Brahmaputra and the SurmaMeghna. This unique geophysical condition constitutes about 46,99,387 ha of diverse inland water areas comprises more than 250 native freshwater fish species (IUCN 2015). Inland fisheries play an important role in the economy of the country in terms of nutrition, income, employment and foreign exchange earnings. More than 11 percent of the total population is engaged in this sector in full time and part time basis for their livelihoods. And fish itself contributes about $60 \%$ of animal protein in the daily dietary requirement of 160 million population of the country (DoF 2018). Inland capture fishery production remarkably declined in the past few decades. Currently, inland capture fishery contributes only $29 \%$ of the country's total fish production which was $63 \%$ in

*Author for correspondence: <sagir@du.ac.bd>, 2Department of Zoology, Jagannath University, Dhaka1100, Bangladesh

(C2020 Zoological Society of Bangladesh DOI: https://doi.org/10.3329/bjz.v48i1.47872 
1983-84 (DoF 2018). This dramatic change in production was due to the continuous habitat destruction, unregulated fishing and aquatic pollution from industrial, domestic and agricultural sources. That is also leading to at least 64 species of freshwater fishes at different categories of threatened with extinction (IUCN 2015).

Nonetheless, description and information of freshwater fishes of Bangladesh are scattered throughout a wide range of publications. The first complete description of freshwater fishes of Bangladesh was compiled by Rahman (1989) which corroborated 260 species including migratory and estuarine species. Siddiqui et al. (2007) described 251 species which is in fact reproduce the previous works.

Published books on diversity of Bangladeshi fishes (Bhuyian 1964, Rahman 2005, Shafi and Quddus 1982, IUCN 2000, IUCN 2015) evident that the information is inconsistent and taxonomy used is not updated.

The accurate identification of fish species is a pivotal component to protect the extant ichthyofaunal biodiversity and to perform regular assessments of local fish faunas for conservation planning. As an alternative to the traditional species identification based on morphological characters, Hebert et al. (2003) has been suggested partial cytochrome c oxidase subunit I (COI) sequences (DNA barcodes) for standardized and routine species identification. The DNA-based barcoding method has been proven to be a valuable molecular tool for species identification and it is accessible to non-specialists (Hebert et al. 2003, Fre'zal and Leblois 2008, Leray et al. 2015). This barcoding technique has been successfully identified ichthyofauna in many geographic regions, such as Australia (Ward et al. 2005), Canada (Hubert et al. 2008), India (Lakra et al. 2015), China (Zhang 2011, Wang et al. 2018), Japan (Zhang and Hanner 2011), Portugal (Costa et al. 2012), Germany (Knebelsberger et al. 2015), Taiwan (Bingpeng et al. 2018, Chang et al. 2017) and Vietnam (Thu et al. 2019), thus enriched the barcode reference library. New specimens and products can confidently be identified by comparing their DNA barcode sequences against this barcode reference library. Although barcodes for almost two-third $(19,000)$ of all described fish species are already available (BOLD 2020), the permanent addition of new barcode data is essential to increase the taxonomic resolution.

Considering the economic importance of inland fishery and the expected richness of the fish fauna and in the absence of an expert-based taxonomy, the first attempt for barcoding of freshwater fishes of Bangladesh was initiated in 2014 and consequently partially published barcode data of 81 small indigenous fish species (SIS) by Ahmed et al. (2019). This paper deals with the molecular characterization of morphologically identified freshwater species of Bangladesh using partial COI gene sequence. 


\section{MATERIAL AND METHODS}

Study area and specimen collection: Fish samples were collected from rivers, haor, baor, beels, floodplain, fish landing centers, fish markets or from the local fishermen during July 2014 to June 2018. Personal fishing was also conducted to collect some rare and non-commercial fish species whenever necessary. Photographs of all the fishes were taken immediately and taxonomic identification of specimens were done following previous reports (Talwar and Jhingran 1991, Rahman 2005, Siddiqui et al. 2007). Immediately after collecting the specimens, tissue samples were excised and stored in $90 \%$ ethanol. Voucher specimens were fixed with $10 \%$ formalin and then transferred to $70 \%$ ethanol solution for preservation. Voucher specimens were transported to Dhaka and deposited in the Professor Kazi Zaker Hussain Museum at the Department of Zoology, University of Dhaka.

DNA barcoding: Genomic DNA was extracted from the muscle tissue samples by the standard Proteinase-K/Phenol-Chloroform-isoamyl alcohol method (Green and Sambrook 2012, Ahmed et al. 2019). The quality and quantity of the extracted DNA was measured using Nanodrop ${ }^{\mathrm{TM}}$ spectrophotometer. Approximately $658 \mathrm{bp}$ was amplified from the 5 ' region of the MT-COI gene using the following primers: FishF1 5'TCAACCAACCACAAAGACATTGGCAC3' and FishR1 5' TAGACTTCTGGGTGGCCAAAGAATCA3' only when failed to amplified the FishF2 5'TCGACTAATCATAAAGATATCGGCAC3' and FishR2 5'ACTTCAGGGTGACCGAAGAATCAGAA3' were used (Ward et al. 2005). For this, $25 \mu \mathrm{l}$ PCR reaction mixtures were prepared which included 17.25-18.75 $\mu \mathrm{l}$ of ultrapure water, $2.5 \mu \mathrm{l}$ of $10 \times \mathrm{PCR}$ buffer, $1.25 \mu \mathrm{l}$ of $\mathrm{MgCl}_{2}(50 \mathrm{mM}), 0.25 \mu \mathrm{l}$ of each primer $(0.01 \mathrm{mM}), 0.125 \mu \mathrm{l}$ of each dNTP $(0.05 \mathrm{mM}), 1 \mu l(0.625 \mathrm{U})$ of Taq polymerase, and 0.5-2.0 $\mu$ l of DNA template. Amplifications were performed using ABI thermal cycler (Thermo Fisher Scientific). The thermal regime consists of an initial step of $2 \mathrm{~min}$ at $95^{\circ} \mathrm{C}$ followed by 35 cycles of $0.5 \mathrm{~min}$ at $94^{\circ} \mathrm{C}, 0.5 \mathrm{~min}$ at $54^{\circ} \mathrm{C}$, and $1 \mathrm{~min}$ at $72^{\circ} \mathrm{C}$, followed in turn by $10 \mathrm{~min}$ at $72^{\circ} \mathrm{C}$ and then held at $4^{\circ} \mathrm{C}$. PCR products were visualized on $1 \%$ agarose gel. The PCR products were purified using PureLink ${ }^{\mathrm{TM}}$ PCR purification kit and sequenced from First BASE Laboratories, Sdn Bhd, Malaysia. All sequences were translated into amino acids to confirm the effectiveness of the sequences and to detect the presence of nuclear DNA pseudo genes, insertions, deletions, or stop codons. Sequences were checked and aligned using Sequencer v5.4.6 and were submitted to GenBank with referred accession numbers. All the data including taxonomic characteristics and GenBank accession numbers were tagged with the voucher specimens preserved at the Professor Kazi Zaker Husain Museum of Department of Zoology, University of Dhaka. 
Bioinformatic and statistical analyses: Bioinformatic analyses of the sequences were performed using CLC Workbench v7.7.1, Mega X, Clustal Omega, and T-Coffee. Base compositions were analyzed using CLC Workbench v7.7.1 and Mega X. Genetic distance and sequence divergences were calculated using the Kimura two parameter (K2P) distance model (Kimura 1980). Neighbor-joining (NJ) trees of K2P distances were created to provide a graphic representation of divergence pattern between species (Saitou and Nei 1987). Bootstrapping was performed in MEGA X (Tamura and Nei 1993) with 1000 replications (Felsenstein 1985). Necessary statistical analyses were performed in Excel 2013 and RStudio.

\section{RESULTS}

A total of 550 tissue samples were collected, during the study period among which 315 COI sequences were obtained (Table 1). Based on morphological and molecular identifications, these samples represented 153 species of 114 genera, 49 families and 16 orders (Table 1). Among the collected species, Order Cypriniformes was recorded as the most diversified fish group in terms of both number of species and individuals observed followed by Perciformes and Siluriformes (Table1). Among the 153 species, 12 fish species were newly recorded in Bangladesh. The average length of all barcode sequences was $652 \mathrm{bp}$ ranging from 352 to $696 \mathrm{bp}$ where $95 \%$ were longer than $600 \mathrm{bp}$. No stop codon, insertion, or deletion was observed in any of the obtained sequences.

Table 1. List of freshwater fish species barcoded along with their GenBank (GB) accession numbers

\begin{tabular}{clllcl}
\hline $\begin{array}{c}\text { SL } \\
\text { No. }\end{array}$ & Order & Family & Species & $\begin{array}{c}\text { No. of } \\
\text { individual }\end{array}$ & $\begin{array}{l}\text { GB Accession } \\
\text { Number }\end{array}$ \\
\hline 1 & Osteoglossiformes & Notopteridae & Chitala chitala & 1 & MF140393 \\
2 & & Notopterus notopterus & 2 & KT346361 KT364757 \\
3 & Elopiformes & Megalopidae & Megalops cyprinoides & 2 & MN171367 MN171368 \\
4 & Anguilliformes & Ophichthidae & Pisodonophis boro & 1 & MG969529 \\
5 & Clupeiformes & Clupeidae & Corica soborna & 2 & KX455892 KY124368 \\
6 & & & Gonialosa manmina & 1 & MH087054 \\
7 & & Tenualosa ilisha & 2 & KX657721 MH230965 \\
8 & & Anodontostoma & 2 & MK878431 MH429338 \\
9 & & Enganda & 2 & MN083101 MH429325 \\
10 & & Setipinna phasa & 3 & MK926759 MN083109 \\
11 & & Coilia ramcarati & & MH311288 \\
& & Coilia dussumieri & 5 & MN083117 MK988524 \\
12 & & & & MN171355 \\
13 & & Pristigasteridae & Ilisha melastoma & 2 & MN200458 MH230984 MN200470 \\
\hline
\end{tabular}




\begin{tabular}{|c|c|c|c|c|c|}
\hline $\begin{array}{l}\text { SL } \\
\text { No. }\end{array}$ & Order & Family & Species & $\begin{array}{c}\text { No. of } \\
\text { individual }\end{array}$ & $\begin{array}{l}\text { GB Accession } \\
\text { Number }\end{array}$ \\
\hline 14 & Gonorynchiformes & Chanidae & Chanos chanos & 1 & MN083123 \\
\hline 15 & Cypriniformes & Nemacheilidae & Acanthocobitis botia & 2 & KT762380 MN013423 \\
\hline 16 & & & $\begin{array}{l}\text { Acanthocobitis } \\
\text { zonalternans }\end{array}$ & 1 & KT762362 \\
\hline 17 & & & Schistura fasciolata* & 1 & KY124367 \\
\hline 18 & & & $\begin{array}{l}\text { Paracanthocobitis } \\
\text { zonalternans }\end{array}$ & 2 & MN200466 MN200467 \\
\hline 19 & & Cyprinidae & $\begin{array}{l}\text { Amblypharyngodon } \\
\text { mola }\end{array}$ & 2 & КT364774 МH087039 \\
\hline 20 & & & Aspidoparia jaya & 2 & MG969527 MG969532 \\
\hline 21 & & & $\begin{array}{l}\text { Barbonymus } \\
\text { gonionotus }\end{array}$ & 1 & KX657718 \\
\hline 22 & & & Barilius barna & 1 & KY124376 \\
\hline 23 & & & Cirrhinus cirrhosus & 1 & KT353104 \\
\hline 24 & & & Cirrhinus reba & 3 & $\begin{array}{l}\text { KX455893 MG969514 } \\
\text { MN083095 }\end{array}$ \\
\hline 25 & & & Crossocheilus latius & 2 & MG969525 MG969531 \\
\hline 26 & & & $\begin{array}{l}\text { Ctenopharyngodon } \\
\text { idella }\end{array}$ & 1 & KX657712 \\
\hline 27 & & & Cyprinus carpio & 3 & $\begin{array}{l}\text { KX657710 KX657711 } \\
\text { MN234111 }\end{array}$ \\
\hline 28 & & & Danio rerio & 1 & MF 170952 \\
\hline 29 & & & Devario aequipinnatus & 3 & $\begin{array}{l}\text { KT364769 KY124372 } \\
\text { KY124375 }\end{array}$ \\
\hline 30 & & & Esomus danricus & 1 & KT364776 \\
\hline 31 & & & Garra nasuta* & 1 & KY124363 \\
\hline 32 & & & Garra sp. & 1 & MF190550 \\
\hline 33 & & & Gibelion catla & 1 & MG969520 \\
\hline 34 & & & $\begin{array}{l}\text { Hypophthalmichthys } \\
\text { molitrix }\end{array}$ & 3 & $\begin{array}{l}\text { KX657713 MH087046 } \\
\text { MF140395 }\end{array}$ \\
\hline 35 & & & Labeo bata & 5 & $\begin{array}{l}\text { KT353105 MG969515 } \\
\text { MN083093 MN083094 } \\
\text { MH087029 }\end{array}$ \\
\hline 36 & & & Labeo calbasu & 1 & KT364767 \\
\hline 37 & & & Labeo gonius & 2 & KX455894 MN200474 \\
\hline 38 & & & Labeo rohita & 4 & $\begin{array}{l}\text { MG969513 MG969519 } \\
\text { MH087049 MF170947 }\end{array}$ \\
\hline 39 & & & Chela cachius & 1 & KT353102 \\
\hline 40 & & & Laubuca laubuca & 1 & KT353103 \\
\hline 41 & & & $\begin{array}{l}\text { Neolissochilus } \\
\text { hexastichus }\end{array}$ & 1 & КT364770 \\
\hline 42 & & & Oreichthys cosuatis & 2 & KX455909 MN013419 \\
\hline 43 & & & Osteobrama cotio & 4 & $\begin{array}{l}\text { KT762359 MN200463 } \\
\text { MN200464 MN200465 }\end{array}$ \\
\hline 44 & & & Pethia conchonius & 4 & $\begin{array}{l}\text { MK988520 MK988542 } \\
\text { KY124379 KY124380 }\end{array}$ \\
\hline 45 & & & Pethia gelius & 2 & MN200473 KT364772 \\
\hline 46 & & & Pethia guganio & 1 & KT762360 \\
\hline
\end{tabular}




\begin{tabular}{|c|c|c|c|c|c|}
\hline $\begin{array}{l}\text { SL } \\
\text { No. }\end{array}$ & Order & Family & Species & $\begin{array}{c}\text { No. of } \\
\text { individual }\end{array}$ & $\begin{array}{l}\text { GB Accession } \\
\text { Number }\end{array}$ \\
\hline 47 & & Cyprinidae & Pethia phutunio & 1 & KT353106 \\
\hline 48 & & & Pethia ticto & 1 & MN083131 \\
\hline 49 & & & Puntius chola & 3 & $\begin{array}{l}\text { KT364771 MN171353 } \\
\text { MN171354 }\end{array}$ \\
\hline $\begin{array}{l}50 \\
51\end{array}$ & & & Systomus sarana & 3 & $\begin{array}{l}\text { KT364773 MH087036 } \\
\text { MN171373 }\end{array}$ \\
\hline 52 & & & Puntius sophore & 1 & KX455895 \\
\hline 53 & & & Puntius terio & 2 & KX455896 MN200455 \\
\hline \multirow[t]{2}{*}{54} & & & Raiamas bola & 1 & KY124369 \\
\hline & & & Rasbora daniconius & 4 & $\begin{array}{l}\text { KT364777 MG280610 } \\
\text { MN013420 MN200472 }\end{array}$ \\
\hline 55 & & & Rasbora rasbora & 1 & MK995091 \\
\hline 56 & & & Salmophasia bacaila & 6 & $\begin{array}{l}\text { MN171372 KT364775 } \\
\text { MG550117 MH087030 } \\
\text { MG550117 MN234103 }\end{array}$ \\
\hline 57 & & & Salmostoma phulo & 1 & KT364758 \\
\hline 58 & & & Securicula gora & 2 & MG969526 MG969533 \\
\hline 59 & & & Tor putitora & 2 & KT762361 KT762379 \\
\hline 60 & & Botiidae & Botia Dario & 5 & $\begin{array}{l}\text { MH087038 MH087045 } \\
\text { MN171346 MN171347 } \\
\text { KY124374 }\end{array}$ \\
\hline 61 & & & Botia lohachata & 3 & $\begin{array}{l}\text { KX455912 MH087044 } \\
\text { MN083135 }\end{array}$ \\
\hline 62 & & & Botia rostrata & 1 & KY124362 \\
\hline 63 & & Cobitidae & Canthophrys gongota & 2 & KX455897 MH087035 \\
\hline 64 & & & $\begin{array}{l}\text { Lepidocephalichthys } \\
\text { annandalei }\end{array}$ & 2 & KY124364 MF140396 \\
\hline 65 & & & $\begin{array}{l}\text { Lepidocephalichthys } \\
\text { guntea }\end{array}$ & 7 & $\begin{array}{l}\text { KT364759 KT364778 } \\
\text { MN013421 MN171348 } \\
\text { MN171349 MN171350 } \\
\text { MN171351 KY124365 }\end{array}$ \\
\hline 66 & & & Pangio pangia & 1 & MF170949 \\
\hline 67 & & Psilorhynchidae & Psilorhynchus balitora & 1 & KY124373 \\
\hline 68 & & & Psilorhynchus sucatio & 1 & MF170951 \\
\hline 69 & Siluriformes & Ailiidae & Ailia coila & 3 & $\begin{array}{l}\text { KT364761 KT364782 } \\
\text { MN083152 }\end{array}$ \\
\hline 70 & & Ariidae & $\begin{array}{l}\text { Osteogeneiosus } \\
\text { militaris }\end{array}$ & 3 & $\begin{array}{l}\text { MH429317 MH429348 } \\
\text { MH230983 }\end{array}$ \\
\hline 71 & & Amblycipitidae & Amblyceps mangois & 1 & KT762370 \\
\hline 72 & & Bagridae & $\begin{array}{l}\text { Batasio } \\
\text { convexirostrum* }\end{array}$ & 1 & KY124366 \\
\hline 73 & & & Mystus bleekeri & 2 & KT364779 MN083144 \\
\hline 74 & & & Mystus cavasius & 3 & $\begin{array}{l}\text { KT762365 KX657719 } \\
\text { MN083157 }\end{array}$ \\
\hline 75 & & & Mystus tengara & 3 & $\begin{array}{l}\text { KT762366 MK988521 } \\
\text { MN083145 }\end{array}$ \\
\hline 76 & & & Mystus vittatus & 1 & KT364780 \\
\hline 77 & & Bagridae & Mystus gulio & 4 & $\begin{array}{l}\text { KX455898 KX455905 } \\
\text { MN083111MK995086 }\end{array}$ \\
\hline
\end{tabular}




\begin{tabular}{|c|c|c|c|c|c|}
\hline $\begin{array}{l}\text { SL } \\
\text { No. }\end{array}$ & Order & Family & Species & $\begin{array}{c}\text { No. of } \\
\text { individual }\end{array}$ & $\begin{array}{l}\text { GB Accession } \\
\text { Number }\end{array}$ \\
\hline 78 & & & Hemibagrus menoda & 2 & KT762363 MG969522 \\
\hline 79 & & & Rama chandramara & 1 & KT762367 \\
\hline 80 & & & Rita rita & 2 & КT364781 KT762374 \\
\hline 81 & & & Sperata aor & 1 & KT762381 \\
\hline 82 & & & Sperata seenghala & 3 & $\begin{array}{l}\text { KT364786 KT762382 } \\
\text { MN171374 }\end{array}$ \\
\hline 83 & & Plotosidae & Plotosus canius & 3 & $\begin{array}{l}\text { KX657716 MK995093 } \\
\text { MN171370 }\end{array}$ \\
\hline 84 & & Chacidae & Chaca chaca & 2 & KX455900 MN083143 \\
\hline 85 & & Clariidae & Clarias batrachus & 1 & KT762385 \\
\hline 86 & & & Clarias gariepinus & 1 & KX657715 \\
\hline 87 & & Schilbeidae & Clupisoma prateri* & 5 & $\begin{array}{l}\text { KT364783 KT762369 } \\
\text { KX455899 MG969517 } \\
\text { MN200476 }\end{array}$ \\
\hline 88 & & & Clupisoma garua & 1 & KX455904 \\
\hline 89 & & & Eutropiichthys vacha & 1 & KT364762 \\
\hline 90 & & & $\begin{array}{l}\text { Neotropius } \\
\text { atherinoides }\end{array}$ & 2 & $\begin{array}{l}\text { KT364763.1 } \\
\text { KT364784 }\end{array}$ \\
\hline 91 & & Sisoridae & Gagata cenia & 2 & KT762384 MG969536 \\
\hline 92 & & & Gagata gagata & 2 & KT364785 MG969523 \\
\hline 93 & & & Bagarius bagarius & 4 & $\begin{array}{l}\text { KT762371 KX455910 } \\
\text { MG969530 MN200478 }\end{array}$ \\
\hline 94 & & & Glyptothorax indicus & 1 & MH087037 \\
\hline 95 & & Erethistidae & Hara jerdoni & 1 & KT762372 \\
\hline 96 & & & Erethistes pusillus & 1 & MG969534 \\
\hline 97 & & Heteropneustidae & Heteropneustes fossilis & 5 & $\begin{array}{l}\text { KT364787 MG969521 } \\
\text { MN083153 MN083154 } \\
\text { MN083155 }\end{array}$ \\
\hline 98 & & Olyridae & Olyra longicaudata & 2 & MF176156 KT762373 \\
\hline 99 & & Siluridae & Ompok bimaculatus & 3 & $\begin{array}{l}\text { KT762368 MH087040 } \\
\text { MN083156 }\end{array}$ \\
\hline 100 & & & Ompok pabda & 3 & $\begin{array}{l}\text { KT364760 KT762383 } \\
\text { MN200457 }\end{array}$ \\
\hline 101 & & & Ompok pabo & 1 & KX455911 \\
\hline 102 & & & Wallago attu & 2 & KX657717 MH087042 \\
\hline 103 & & Pangasiidae & $\begin{array}{l}\text { Pangasianodon } \\
\text { hypophthalmus }\end{array}$ & 1 & MF373123 \\
\hline 104 & Batrachoidiformes & Batrachoididae & $\begin{array}{l}\text { Batrachomoeus } \\
\text { trispinosus* }\end{array}$ & 3 & $\begin{array}{l}\text { MN234104 MN234105 } \\
\text { MN234107 }\end{array}$ \\
\hline 105 & Cyprinodontiformes & Poeciliidae & Poecilia reticulata & 1 & MN083133 \\
\hline 106 & Syngnathiformes & Syngnathidae & Microphis deocata & 1 & KT762375 \\
\hline 107 & Synbranchiformes & Mastacembelidae & Macrognathus aral & 6 & $\begin{array}{l}\text { MK995083 MN083138 } \\
\text { MN083148 MN083149 } \\
\text { KT762377 MF170946 }\end{array}$ \\
\hline 108 & & Mastacembelidae & $\begin{array}{l}\text { Macrognathus } \\
\text { pancalus }\end{array}$ & 4 & $\begin{array}{l}\text { KT762378 MH087034 } \\
\text { MN200459 MN200460 }\end{array}$ \\
\hline
\end{tabular}




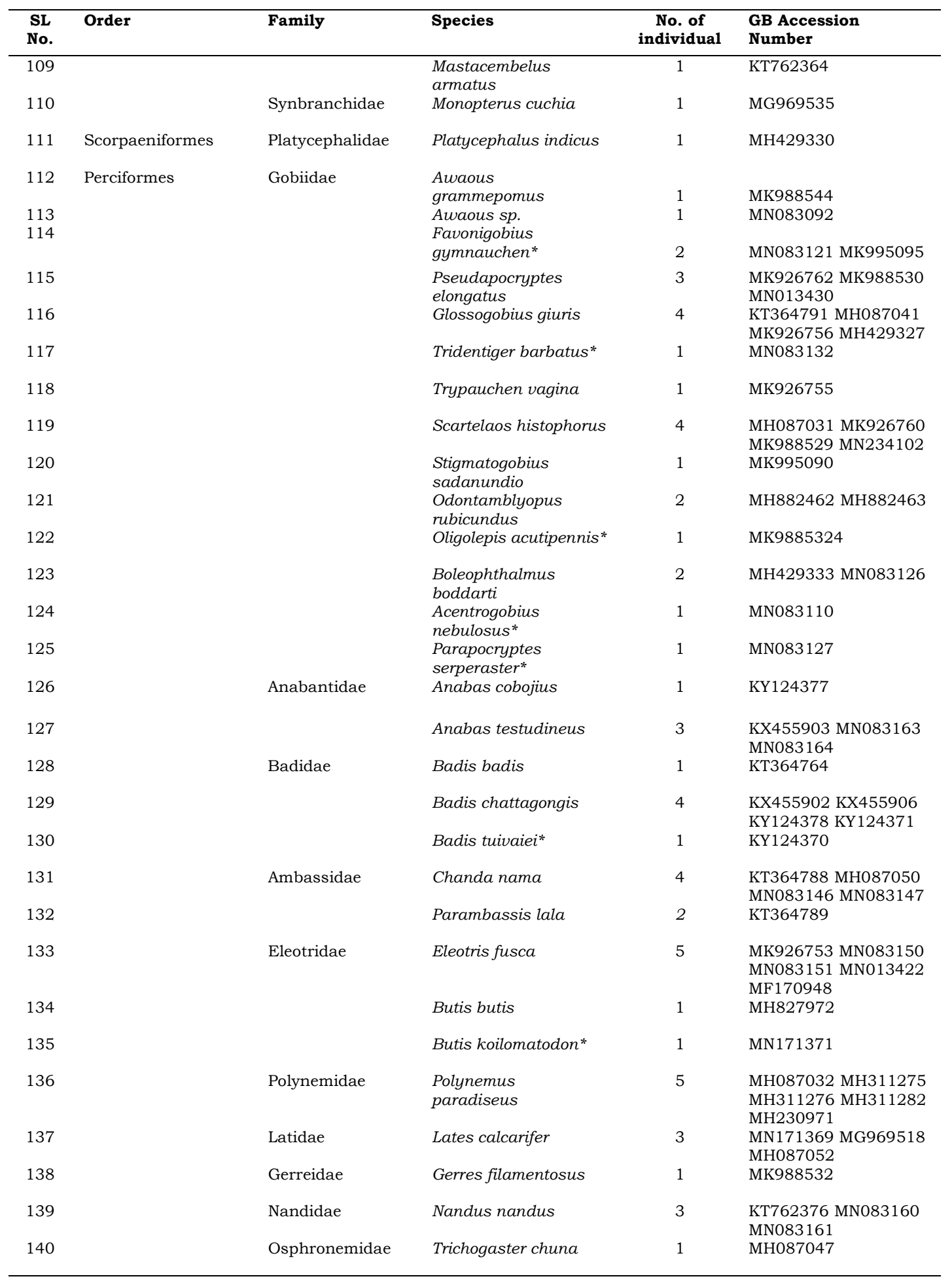




\begin{tabular}{|c|c|c|c|c|c|}
\hline $\begin{array}{l}\text { SL } \\
\text { No. }\end{array}$ & Order & Family & Species & $\begin{array}{c}\text { No. of } \\
\text { individual }\end{array}$ & $\begin{array}{l}\text { GB Accession } \\
\text { Number }\end{array}$ \\
\hline 141 & & & Trichogaster fasciata & 1 & MH087051 \\
\hline 142 & & & Trichopsis vittata & 3 & $\begin{array}{l}\text { KT364765 КT364768 } \\
\text { KT364792 }\end{array}$ \\
\hline 143 & & Mugilidae & Rhinomugil corsula & 5 & $\begin{array}{l}\text { KT364790 MG969528 } \\
\text { MN083165 MN171356 } \\
\text { MN171357 }\end{array}$ \\
\hline 144 & & & Mugil cephalus & 1 & MK988536 \\
\hline 145 & & Cichlidae & Oreochromis niloticus & 1 & KX657714 \\
\hline 146 & & Channidae & Channa marulius & 2 & KX808573 MG969516 \\
\hline 147 & & & Channa gachua & 1 & KT364793 \\
\hline 148 & & & Channa punctata & 1 & KT762386 \\
\hline 149 & & & Channa striata & 1 & KT762387 \\
\hline 150 & Beloniformes & Adrianichthyidae & Oryzias javanicus* & 1 & MF170950 \\
\hline 151 & & Belonidae & Xenentodon cancila & 1 & MH087053 \\
\hline 152 & & Zenarchopteridae & $\begin{array}{l}\text { Zenarchopterus } \\
\text { ectuntio }\end{array}$ & 1 & MK988518 \\
\hline 153 & Tetraodontiformes & Tetraodontidae & Leiodon cutcutia & 4 & $\begin{array}{l}\text { MK926757 MN200461 } \\
\text { MN200462 MF140394 }\end{array}$ \\
\hline
\end{tabular}

Table 2. Genetic divergence (\%K2P distance) of freshwater fishes within various taxonomic levels

\begin{tabular}{lcllll}
\hline Level & Sample size & Mean & Minimum & Maximum & SE \\
\hline Species & 145 & 0.38 & 0.00 & 2.93 & 0.01 \\
Genus & 105 & 7.02 & $0.00^{*}$ & 28.67 & 0.02 \\
Family & 44 & 12.75 & 0.57 & 22.39 & 0.03 \\
Order & 13 & 18.68 & 5.95 & 26.18 & 0.04 \\
\hline
\end{tabular}

*Single sequence

The lack of stop codons in these sequences indicates that they are functional mitochondrial COI sequences, together with the fact that each of the amplified sequence was about $658 \mathrm{bp}$ in length. Hence, it suggests that Nuclear DNA Sequences Originating from Mitochondrial DNA Sequences (NUMTs) were not sequenced, as vertebrate NUMTS are typically less than $600 \mathrm{bp}$ (Zhang and Hewitt 1996).

The sequence analysis indicated that the average nucleotide frequencies to be A: $25.20 \%$, T:29.80\%, G: $17.70 \%$ and C: $27.30 \%$ (Fig. 1).The base composition analysis for the COI sequence showed that the average $\mathrm{T}$ content was the highest and the average G content was the lowest; the AT content (55.0\%) was higher than 


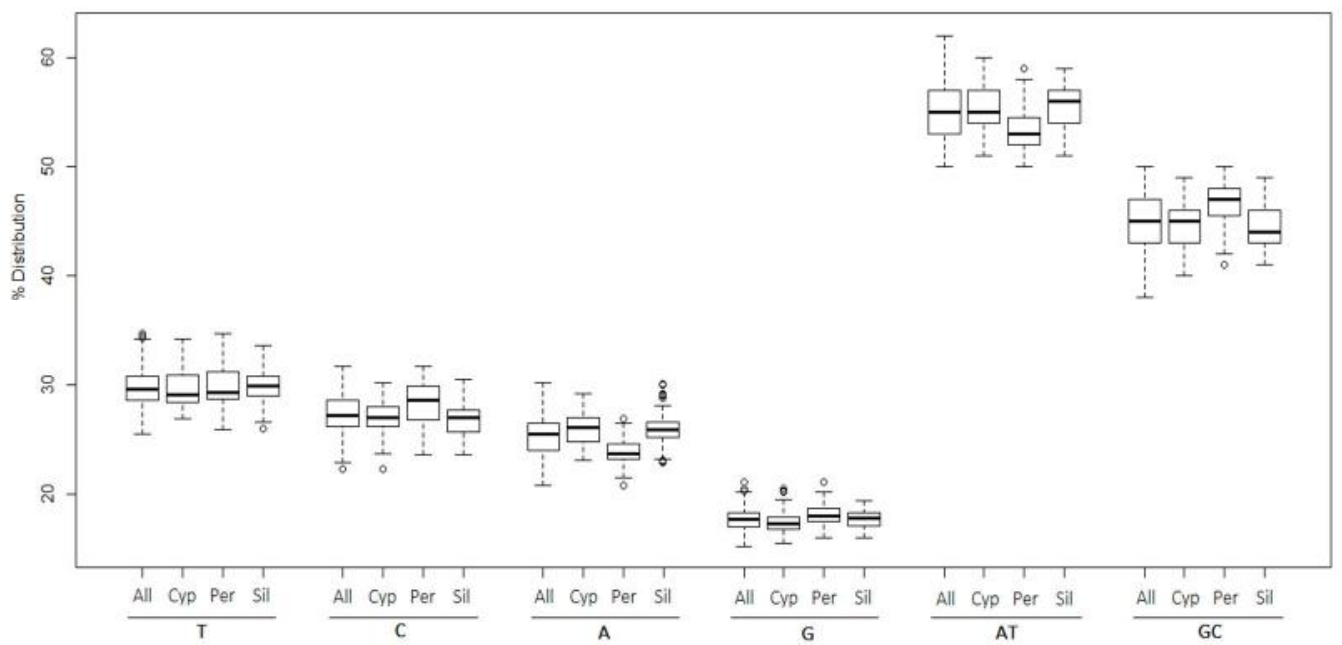

Fig. 1. Composition of the nucleotides in the sequenced COI region of freshwater fishes of Bangladesh.

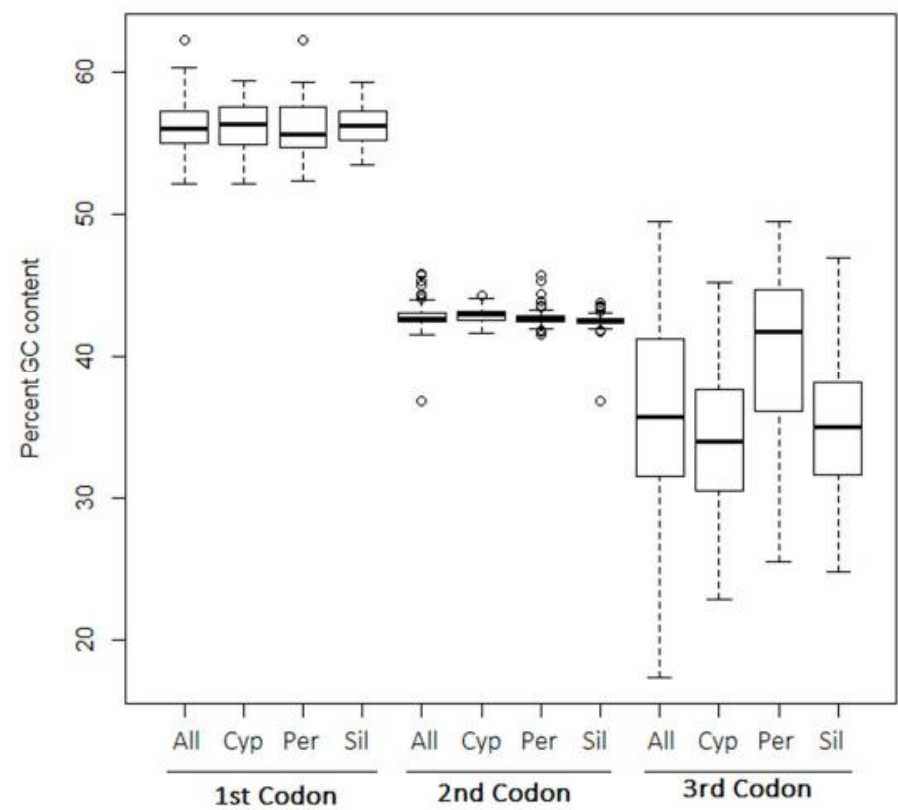

Fig. 2. Percent (\%) GC content at different codon positions in the sequenced COI region of freshwater fishes of Bangladesh. 


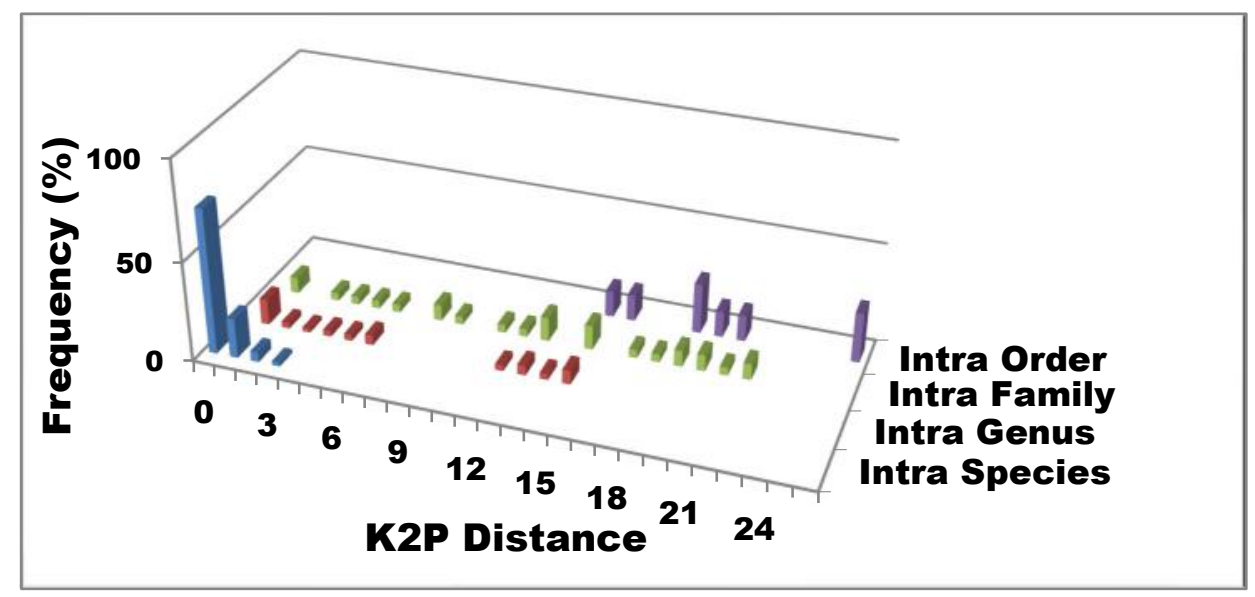

Fig. 3. Distribution of K2P distances (percentage) within different taxonomic categories.

the GC content (45.0\%). The GC contents at the first, second and third codon positions for all fish were $56.09 \%, 42.78 \%$ and $36.02 \%$ respectively (Fig. 2). At the first codon position, the usage of $\mathrm{T}(19.10 \%)$ was the lowest, and the usages of the other bases were C: $25.40 \%, \mathrm{~A}: 24.80 \%$ and G: $30.60 \%$. At the second codon position, the content of $\mathrm{T}(41.80 \%)$ was highest, and the contents of the other bases were C: $28.10 \%$, A: $15.50 \%$ and G: $14.70 \%$. At the third codon position, the base usage was T $(28.40 \%), \mathrm{C}(28.20 \%)$, A $(35.60 \%)$ and G $(7.80 \%)$; the G content was the lowest, exhibiting a clear pattern of anti-G bias. The average genetic distance within species, genus, family and order were $0.38 \pm 0.01 \%, 7.02 \pm 0.02 \%$, $12.75 \pm 0.03 \%$ and $18.68 \pm 0.04 \%$, respectively (Table 2). The NJ tree of 315 generated sequences including 153 species is presented in Figure 4.

Order Cypriniformes: This order includes many of the most important forage and food fish. A total of 112 samples were sequenced belonging to five families, 37 genera and 54 species. The overall mean nucleotide base frequencies observed for these sequences were- T: $29.70 \%, \mathrm{C}: 27.0 \%, \mathrm{~A}: 25.80 \%$ and G: $17.50 \%$. The AT content (55.50\%) was higher than the GC content $(44.50 \%)$. The GC contents at the first, second and third codon positions were $49.10 \%, 48.20 \%$ and $44.40 \%$ respectively. The NJ tree clearly distinguished all the species (Fig. 4).

Order Perciformes: A total of 72 samples were sequenced belonging to 12 families, 27 genera and 33 species. The overall mean nucleotide base frequencies observed for these sequences were T: $29.90 \%$, C: $28.20 \%$, A: $23.80 \%$ and G: $18.10 \%$. The AT content (53.70\%) was higher than the GC content $(46.30 \%)$. The GC contents at the first, second and third codon positions were $56.70 \%, 42.70 \%$ 
and $39.50 \%$, respectively. In the NJ tree, most of the specimens belonging to the same species were clustered together bolstering the prior taxonomic assignment based on morphology (Fig. 4).

Order Siluriformes: A total of 76 samples were sequenced belonging to 14 families, 25 genera and 36 species. The overall mean nucleotide base frequencies observed for these sequences were- T: $29.70 \%, \mathrm{C}: 26.90 \%, \mathrm{~A}: 25.60 \%$ and G: $17.70 \%$. The AT content (55.30\%) was higher than the GC content $(44.70 \%)$. The GC contents at the first, second and third codon positions were $56.70 \%$, $42.70 \%$ and $38.10 \%$ respectively.

\section{DISCUSSION}

DNA barcoding could be considered as a global bio-scanner for rapid and authentic identification of organisms using the partial sequence of mitochondrial COI gene. Barcoding has clearly discriminated freshwater fish species from around the globe including Australia, Canada, India, Thailand, Germany (Ward et al. 2005, Hubert et al. 2008, Lakra et al. 2015, Panprommin et al. 2019, Knebelsberger et al. 2014). Here, we have complied the COI sequence profile of freshwater fishes collected from the different inland waters of Bangladesh and confirmed the efficacy of barcoding to identify these species. Barcodes were generated for 153 species of belonging to 114 genera and 49 families and 16 orders (Table 1). We observed no insertions/ deletions or codon stops after translating the nucleotide sequences, supporting the view that all of the amplified sequences denote functional mitochondrial COI sequences. Moreover, average length of the amplified sequences was larger than $650 \mathrm{bp}$, the limit typically observed for nuclear DNA sequences originating from mtDNA (NUMTs) (Gunbin et al. 2017). All of these species were differentiable based on the individual COI barcodes. Hence, this study has strongly validated the efficiency of COI barcodes for identifying fish species.

The base composition analysis of the COI sequences revealed that AT content $(55.0 \%)$ to be higher than GC content (45.0\%), similar to the patterns were observed in Australian (Ward et al. 2005), Canadian (Steinke et al. 2009) and Cuban fish species (Lara et al. 2010). The GC contents in the first, second and third codon positions were $56.09 \%, 42.78 \%$ and $36.02 \%$, respectively (Fig. 2). At the first codon position, the usage of $\mathrm{G}(19.00 \%)$ was the lowest, and the usages of the other bases were $23.9 \%, 32.6 \%$ and $24.00 \%$ for C, A and T, respectively. At the second codon position, the content of $\mathrm{T}(31.00 \%)$ was highest, and the contents of the other bases were $25.7 \%, 23.1 \%$ and 20.0 for C, A and G, respectively. At the third codon position, the base usage was- T: $33.00 \%, \mathrm{C}: 32.0 \%$, 


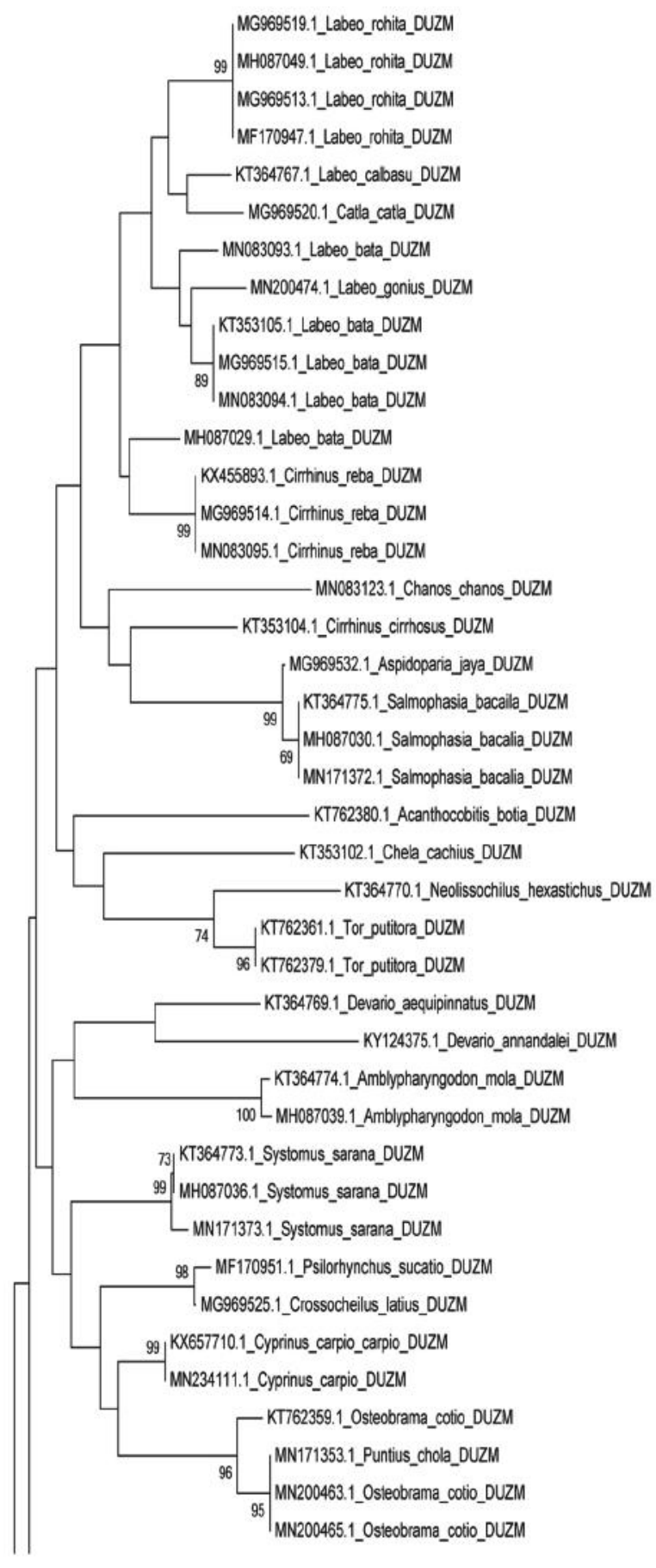

A

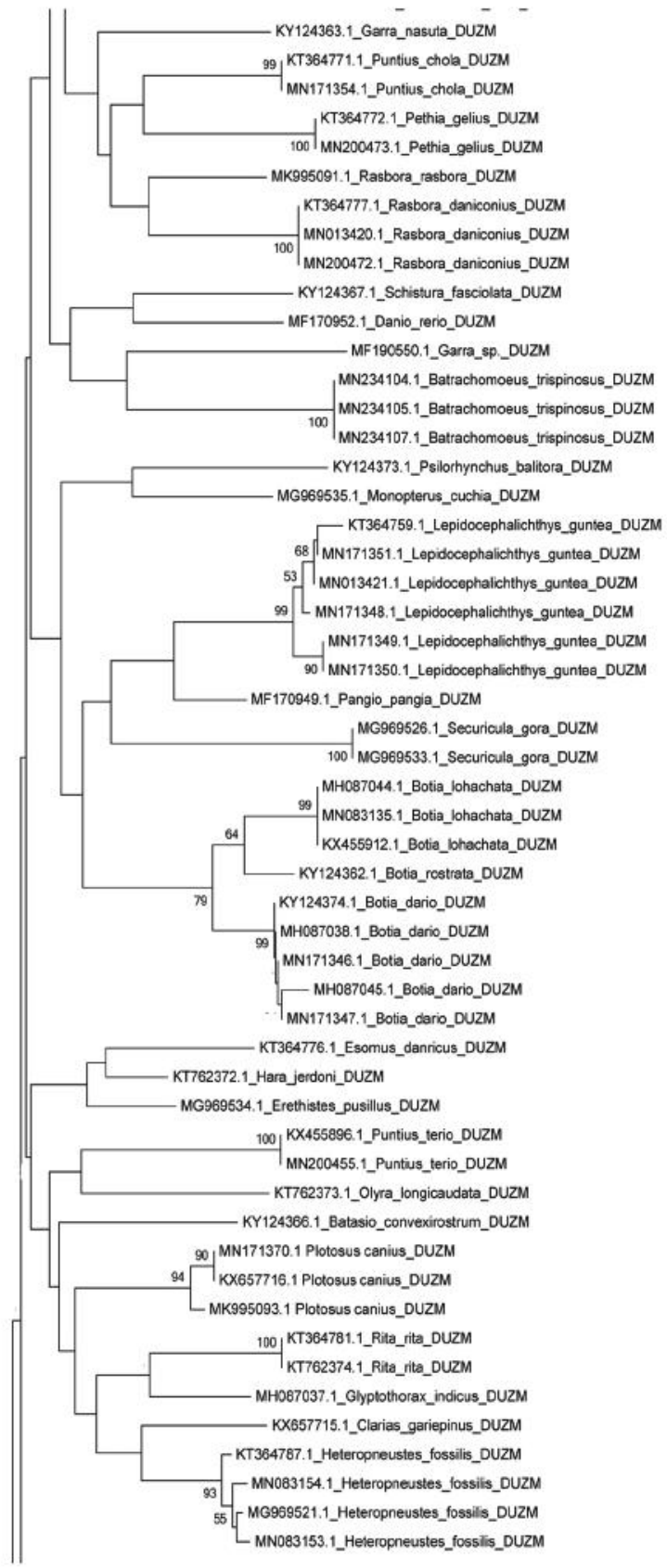

Fig. 4. Neighbor-joining (NJ) tree of freshwater fishes using K2P distances. 


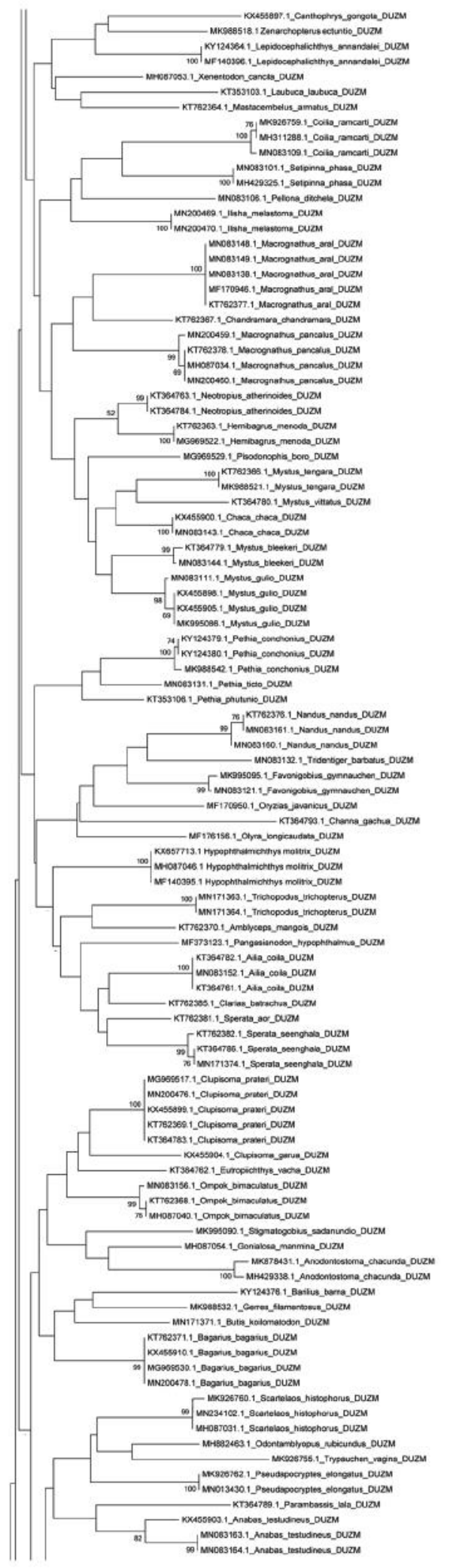

$\mathrm{C}$

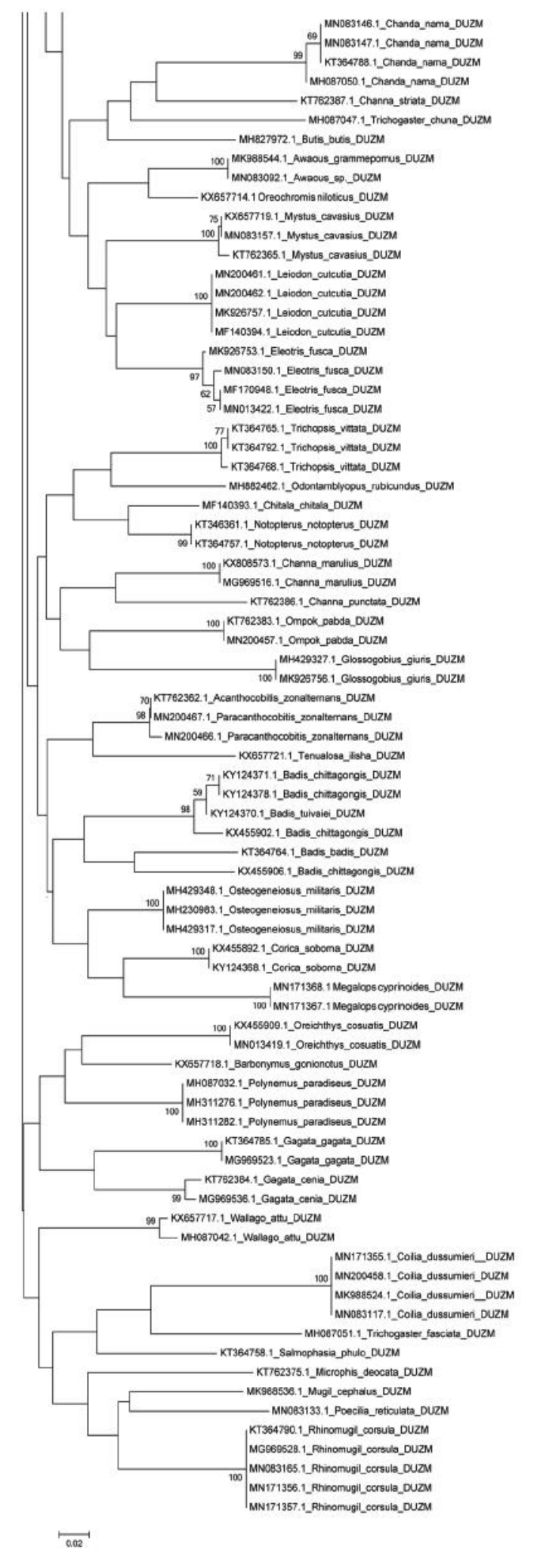

$\mathrm{D}$

Fig. 4. Neighbor-joining (NJ) tree of freshwater fishes using K2P distances(Contd.) 
A:20.3\% and G:14.3\%. There was a significantly higher overall GC content in the 153 species and this difference was attributable to the GC content at the $2^{\text {nd }}$ and the $3^{\text {rd }}$ codon base. The pattern of \%GC content at different codons for all the fishes was invariably $1^{\text {st }}>2^{\text {nd }}>3^{\text {rd }}(p<0.005)$ (Fig. 2$)$.

Kimura 2-parameter distance values of $7.02 \pm 0.02 \%, 12.75 \pm 0.03 \%$ and $18.68 \pm 0.04 \%$ were obtained for within genus, within family and within order respectively (Table 2, Fig. 3). The pairwise genetic distance values were increasing at higher taxonomic levels as expected and consistent with the previous studies that supports the significant change in genetic divergence at the species boundaries (Hubert et al. 2008, Lakra et al. 2015, Ahmed et al. 2019). In this study, the average K2P distance within species was $0.38 \%$, compared with $7.02 \%$ for within genera. The mean interspecific distance was found to be 18 -fold higher than the mean intraspecific distance. More than 20-fold difference was observed in the freshwater fishes commonly encountered in the Australian, Canadian and Indian freshwater fishes (Ward et al. 2005, Hubert et al. 2008, Lakra et al. 2015). This finding corresponds to the DNA barcoding principle that interspecific divergence sufficiently outscores intraspecific divergence.

The accuracy of species identification through DNA barcoding mostly depends on both interspecific and intraspecific divergence. In our study, the average genetic distance within species was found $0.38 \pm 0.01 \%$. Mean intraspecific genetic distance was calculated as $<1 \%$ in previous studies; Hubert et al. (2008) found $0.30 \%$ Lakra et al. (2015) $0.25 \%(0-0.82 \%)$ for the freshwater fishes. Phylogenetic relationships of barcoded species were shown in NJ tree (Fig. 4). Each species was associated with a specific DNA barcode cluster and the relationship among these species was clearly revealed. Closer species in terms of genetic divergence, were clustered at the same nodes and the distance between the terminal branches of the $\mathrm{NJ}$ tree widened as they got more distinct.

In some cases, deviations were observed. For example, out of five sequences of Labeo bata two form different cluster, one (MH087029.1) with Cirrhinus reba and another (MN083093.1) showed more close relationship with Labeo gonius (Fig. 4). We know that L. bata is widely used as an aquaculture species and its induced breeding performed almost all hatcheries of the country. Not only this, its fry and fingerlings are released in the natural habitat. So, it not unlikely to be its hybrid population in natural habitats. Moreover, among the three sequences of Anabas testudineus, one sequence deviated from the rest with $83 \%$ bootstrap value and we suspect this an exotic variety (Thai koi) which is now available everywhere in the country. Channa orientalis was documented in Bangladesh (Siddiqui et al. 2007, IUCN 2015) which is an endemic species of Sri Lanka, and it often misidentified as C. gachua (Tanomtong et al. 2014). The major 
morphological difference between the two species is that C. gachua has ventral fins and $C$. orientalis lack of ventral fin. We could not find any specimen of $C$. orientalis, and this led us to believe that $C$. orientalis not existing in Bangladesh and all the older reports are pertaining to C. gachua (Ahmed et al. 2018, ConteGrand et al. 2017). Macrognathus of Bangladesh having been referred by virtually all authors to M. aculeatus (Rahman 2005, Siddiqui et al. 2007). M. aculeatus body usually with 14-17 oblique dark bars whereas, M. aral body typically with two or more broad pale longitudinal bands of varying width extending its entire length, never with oblique bars (Roberts 1980). Our five generated sequences of collected species showed high similarity with GB pre-existing sequences and we thus confirm the presence of $M$. aral instead of $M$. aculeatus. Loaches are another diverse group of fishes with very confusing characteristics. At least three new records (Garra nasuta, Schistura fasciolata, Botia rostrata) have been confirmed in this study and we presume that some new species/records are still to be explored under the genus Lepidocephalichthys, Botia, or Garra. A taxonomic revision on taxa of this group is urgently needed for their biodiversity conservation.

The present study revealed that DNA barcoding has been successful in identifying the freshwater fishes of Bangladesh. We have barcoded 153 species of freshwater fishes and these barcode data confirms the 12 new records from Bangladesh. When traditional morpho taxonomy does not work, this molecular tool is effective for species identification, particularly with specimens that are damaged, incomplete, or morphologically distinct stages. Nevertheless, DNA barcoding also has its limitations too. Therefore, DNA barcoding can serve as a complementary tool for species identification, but it cannot replace the traditional morpho-taxonomy. Through this study, a reliable DNA barcode reference library for Bangladeshi freshwater fish was established, which could be used to assign fish species by screening sequences against it in the future. This could enhance to achieve better monitoring, conservation, and management of fisheries in this overexploited country.

Acknowledgements: We acknowledge the financial support from the USAID Trust Fund and the Ministry of Agriculture, Government of the Peoples Republic of Bangladesh, as a Competitive Research Grant (Grant no. CRG 471, 2017-2018) to the first author through the National Agricultural Technology Program-Phase II Project (NATP-2) from Bangladesh Agricultural Research Council (BARC), Dhaka, Bangladesh. We are also thankful to Zarif Hossain, Nusrat Jahan Sanzida, J.B.M. Aysha Akter, Nishat Jahan Chowdhury, Tonmoy Saha, Sumaiya Salam, Tamim Afrin, Anindita Barua and Noor Aida Arfin for their support during this research. 


\section{LITERATURE CITED}

AHMED, M.S., CHOWDHURY, M.M.K. and NAHAR, L. 2019. Molecular characterization of small indigenous fish species (SIS) of Bangladesh through DNA barcodes. Gene. 684: 53-57.

AHMED, M.S., DINA, S.R., NAHAR, L., ISLAM, N.N. and REZA, A.H. 2018. Molecular characterization of Channa species from Bangladesh based on Cytochrome c Oxidase Subunit I (COI) gene. FishTaxa. 3(4): 87-93.

BHUIYAN, A. L. 1964. Fishes of Dacca. Asiatic Society of Pakistan, Dacca, 148pp.

BINGPENG, X., HESHAN, L., ZHILAN, Z., CHUNGUANG, W., YANGUO, W. and JIANJUN, W. 2018. DNA barcoding for identification of fish species in the Taiwan Strait. PLOS One. 13(6): 13(6): e0198109. doi.org/10.1371/journal.pone.0198109.

BOLD 2020. The Barcode of Life Data System (http://www.boldsystems.org).

CHANG, C.H., SHAO, K.T., LIN, H.Y., CHIU, Y.C., LEE, M.Y., LIU, S. H. and LIN, P. L. 2017. DNA barcodes of the native ray-finned fishes in Taiwan. Mol. Ecol. Resour. 17(4): 796-805.

CONTE-GRAND C., BRITZ R., DAHANUKAR N., RAGHAVAN R., PETHIYAGODA R. and TAN H.H. 2017. Barcoding snakeheads (Teleostei, Channidae) revisited: Discovering greater species diversity and resolving perpetuated taxonomic confusions. PLOS One. 12(9): e0184017.

COSTA, F.O., LANDI, M., MARTINS, R., COSTA, M.H., COSTA, M.E., CARNEIRO, M. and CARVALHO, G.R. 2012. A ranking system for reference libraries of DNA barcodes: application to marine fish species from Portugal. PLOS One. 7(4): e35858. doi:10.1371/journal.pone.0035858

DoF (Department of Fisheries). 2018. Yearbook of Fisheries Statistics of Bangladesh, 2017-18. Fisheries Resources Survey System (FRSS), Department of Fisheries. Bangladesh: Ministry of Fisheries, 2018. Volume 35:129pp.

FELSENSTEIN, J. 1985. Confidence limits on phylogenies: an approach using the bootstrap. Evolution. 39(4): 783-791.

FRE'ZAL, L. and LEBLOIS, R. 2008. Four years of DNA barcoding: current advances and prospects. Infect. Genet. and Evol.8(5):727-36.

GREEN, M. R and SAMBROOK, J. 2012. A laboratory manual. Cold Spring Harbor. 178pp.

GUNBIN, K., PESHKIN, L., POPADIN, K., ANNIS, S., ACKERMANN, R.R. and KHRAPKO, K. 2017. Data on the time of integration of the human mitochondrial pseudogenes (NUMTs) into the nuclear genome. Data in brief. 13: 536-544.

HEBERT, P.D., CYWINSKA, A., BALL, S.L. 2003. Biological identifications through DNA barcodes. Proc. Royal Soc. B. 270(1512): 313-21.

HUBERT, N., HANNER, R., HOLM, E., MANDRAK, N.E., TAYLOR, E., BURRIDGE, M. and ZHANG, J. 2008. Identifying Canadian freshwater fishes through DNA barcodes. PLOS One. 3(6): 19.e2490 doi:10.1371/journal.pone.0002490 
IUCN Bangladesh. 2000. Red Book of Threatened Fishes of Bangladesh. IUCN- The World Conservation Union. Xii+116PP.

IUCN Bangladesh. 2015. Red List of Bangladesh Volume 5: Freshwater Fishes. IUCN, International Union for Conservation of Nature, Bangladesh Country Office, Dhaka, Bangladesh. xvi+360.

KIMURA, M. 1980. A simple method for estimating evolutionary rates of base substitutions through comparative studies of nucleotide sequences. J. Mol. Evol. 16:111-120.

KNEBElSBERGER, T., DUNZ, A. R., NEUMANN, D. and GEIGER, M. F. 2015. Molecular diversity of Germany's freshwater fishes and lampreys assessed by DNA barcoding. Mol. Ecol. Resour. 15(3): 562-572.

LAKRA, W.S., SINGH, M., GOSWAMI, M., GOPALAKRISHNAN, A., LAL, K.K., MOHINDRA, V., SARKAR, U.K., PUNIA, P.P., SINGH, K.V., BHATT, J.P. and AYYAPPAN, S.2015. DNA barcoding Indian freshwater fishes. Mitochondrial DNA Part A, 27(6): 4510-4517. DOI: 10.3109/19401736.2015.1101540

LARA, A., PONCE DE LEÓN, J.L., RODRIGUEZ, R., CASANE, D., COTE, G., BERNATCHEZ, L., and GARCÍA-MACHADO, E.R.I.K. 2010. DNA barcoding of Cuban freshwater fishes: evidence for cryptic species and taxonomic conflicts. Mol. Ecol. Resour. 10(3): 421-430.

LERAY, M., KNOWLTON, N. 2015. DNA barcoding and metabarcoding of standardized samples reveal patterns of marine benthic diversity. Proc. Natl. Acad. Sci. 112(7):2076-81.

PANPROMMIN, D., SOONTORNPRASIT, K., TUNCHAROEN, S., PITHAKPOL, S. and KEEREELANG, J. 2019. DNA barcodes for the identification of species diversity in fish from Kwan Phayao, Thailand. J. Asia-Pac Biodiv. 12(3):382-389.

RAHMAN A.K.A. 1989. Freshwater Fishes of Bangladesh, $1^{\text {st }}$ ed., Zool. Soc. Bangladesh, Dhaka, Bangladesh.364pp.

RAHMAN A.K.A. 2005. Freshwater Fishes of Bangladesh, $2^{\text {nd }}$ ed., Zool. Soc. Bangladesh, Dhaka, Bangladesh. xviii+394pp.

ROBERTS, T. R. 1980. A Revision of the Asian Mastacembelid Fish Genus. American Society of Ichthyologists and Herpetologists (ASIH). Copeia.1980(3): 385-391

SAITOU, N. AND NEI, M. 1987. The neighbor-joining method: a new method for reconstructing phylogenetic trees. Mol. Biol. and Evol. 4(4):406-425.

SHAFI, M., and QUDDUS, M.M.A. 1982. Bangladesher Mothsho Shampad (In Bengali). Bangla Academy, Dhaka. 444pp.

SIDDIQUI, K.U., ISLAM, M.A., KABIR, S.M.H., AHMAD, M., AHMED, A.T.A., RAHMAN, A.K.A., HAQUE, E.U., AHMED, Z.U., BEGUM, Z.N.T., HASSAN, M.A., KHONDKER, M. AND RAHMAN, M.M. (eds.) 2009. Encyclopedia of Flora and Fauna of Bangladesh, Vol. 23 Freshwater Fishes. Asiatic Society of Bangladesh, Dhaka.300pp.

STEINKE, D., ZEMLAK, T.S., BOUTILLIER, J.A., and HEBERT, P.D. 2009. DNA barcoding of Pacific Canada's fishes. Mar.Biol. 156(12): 2641-2647. 
TALWAR, P.K. and JHINGRAN, A.G. 1991. Inland fisheries of India and adjacent countries. Vol. I \& II: 1-1158.

TAMURA, K. and NEI, M. 1993. Estimation of the number of nucleotide substitutions in the control region of mitochondrial DNA in humans and chimpanzees. Mol. Biol. and Evol. 10(3): 512526.

TANOMTONG, A., SUPIWONG W., JEARRANAIPREPAME P., KHAKHONG S., KONGPIRONCHUEN C., GETLEKHA N. 2014. A new natural autotetraploid and chromosomal characteristics of dwarf snakehead fish, Channa gachua (Perciformes, Channidae) in Thailand. Cytologi.79(1): 15-27.

THU, P. T., HUANG, W. C., CHOU, T. K., VAN QUAN, N., VAN CHIEN, P., LI, F. and LIAO, T. Y. 2019. DNA barcoding of coastal ray-finned fishes in Vietnam. PLOS One. 14(9): e0222631. https://doi.org/10.1371/journal.pone.0222631

WANG, L., WU, Z., LIU, M., LIU, W., ZHAO, W., LIU, H., and YOU, F.2018. DNA barcoding of marine fish species from Rongcheng Bay, China. Peer J. 6: e5013.

WARD, R.D., ZEMLAK, T.S., INNES, B.H., LAST, P.R. and HEBERT, P.D. 2005. DNA barcoding Australia's fish species. Philos. Trans. R. Soc. B. 360: 1847-1857.

ZHANG, D. X., and HEWITT, G. M. 1996. Nuclear integrations: challenges for mitochondrial DNA markers. Trends Ecol. Evol. 11(6): 247-251.

ZHANG, J. 2011. Species identification of marine fishes in China with DNA barcoding. Evid.-Based Complementary Altern. Med. 2011(978253):10 pp.doi:10.1155/2011/978253

ZHANG, J. B. AND HANNER, R. 2011. DNA barcoding is a useful tool for the identification of marine fishes from Japan. Biochem. Syst. and Ecol., 39(1): 31-42

(Manuscript received on 15 May, 2020 revised on 29 May, 2020) 